\title{
Selfishness in Device-to-Device Communication Underlaying Cellular Networks
}

\author{
Haoming Zhang, Yong Li, Depeng Jin and Sheng Chen
}

\begin{abstract}
In a device-to-device (D2D) communication underlaying cellular network, user equipments are required to operate cooperatively and unselfishly to transmit data as relays. However, most users behave in a more or less selfish way, which makes user selfishness a key factor that affects the performance of the whole communication system. We focus on the impact of node selfishness on D2D communications. By separating the user selfishness into two types in accordance with two D2D transmission modes - connected D2D transmission and opportunistic D2D transmission, we propose a time-varying graph model that characterizes the impacts of both individual and social selfishness on D2D communications. Simulation results obtained under the realistic networking settings indicate that the interaction between connected and opportunistic selfishness worsens the impairment caused by individual selfishness, while the harmful interaction caused by social selfishness can be alleviated.
\end{abstract}

\section{INTRODUCTION}

With the increasing demand for content downloading by mobile devices, cellular direct transmission may be unable to meet the downloading demand [1]-[3]. Hence, 3GPP has integrated the device-to-device (D2D) communication as an underlay to the next generation cellular network known as LTE-A. Utilizing the physical proximity of mobile devices and local good channel conditions [1], [2], D2D communications require base stations (BSs) to distribute cellular resources to communicating user equipment (UE) pairs [4]. With the allocated resources, an UE can not only help to forward data from a BS or other UE to another UE, but also temporally stores some data in its buffer and wait for contact opportunities to send them [5], which compose the two D2D transmission modes known as connected D2D and opportunistic D2D transmissions [6]. In order to benefit the whole community, communicating devices are required to devote some of their own resources such as power, CPU occupation and buffer to the D2D protocol in a unselfish way [7].

In the real world, mobile users have the instinctive and indispensable nature - selfishness [8], which is the unavoidable result of many practical factors, such as finite energy, limited storage, valuable CPU occupation and security considerations. Unfortunately, current researches have assumed cooperative and selfless UEs and fail to consider selfishness in D2D

H. Zhang, Y. Li and D. Jin are with Tsinghua National Laboratory for Information Science and Technology, Department of Electronic Engineering, Tsinghua University, Beijing 100084, China

S. Chen is with Electronics and Computer Science, University of Southampton, Southampton SO17 1BJ, UK. He is also with King Abdulaziz University, Jeddah 21589, Saudi Arabia

This work is supported by the National Basic Research Program of China (973 Program) (No. 2013CB329105) and National Nature Science Foundation of China (No. 61301080, No. 91338203, and No. 91338102). systems, which may lead to overestimation of the system performance and misinterpretation of the relevant properties. Obviously, if most users are unwilling to participate in D2D transmission, the resources cannot be utilized sufficiently, and a D2D underlaying cellular system will be unable to operate successfully. Consequently, user selfishness, which has not been studied by the existing works but is intrinsic and crucial to $\mathrm{D} 2 \mathrm{D}$ systems, has to be understood in order to objectively evaluate the performance of D2D systems.

However, it is difficult to quantify the impacts of this psychical attribute of the cellular users. As an individual, an UE tends to show unwillingness when required to selflessly forward or store data for other users. This type of selfishness is called individual selfishness. As a member of a community, an UE is more willing to act as relay for the members of the same community, but may be unwilling to do so for the users outside the community. This type of selfishness is named social selfishness. Both types of selfishness should be characterized and, moreover, their interplay in different D2D transmission modes should be considered. Against this background, our contributions are summarized as follows.

- We are the first to investigate the impacts of selfishness on D2D underlaying cellular networks, by defining two new selfishness modes, known as D2D connected selfishness and D2D opportunistic selfishness, in accordance with the two D2D transmission modes.

- We propose a time-varying graph model capable of revealing the impacts of both individual and social selfishness on the two D2D transmission modes. With this model, D2D connected selfishness and D2D opportunistic selfishness are studied both separately and jointly in terms of both individual selfishness and social selfishness.

- We implement extensive simulations with realistic human selfishness and network settings based on this model, and draw valuable conclusions regarding exasperated impairments caused by interaction between the two D2D selfishness modes. We also discuss the differences between individual and social D2D selfishness.

\section{System Overview And Motivation}

Fig. 1 depicts a D2D underlaying cellular network with selfish UEs. Since UEs are naturally mobile nodes with varying positions and access states, we use "time frame" to loosely mark a system time period within which the access states and physical relationships of all the nodes remain nearly constant, and Fig. 1 includes two different time frames, $t_{2}$ and $t_{4}$. The orange hexagons indicate the approximate coverage areas of 


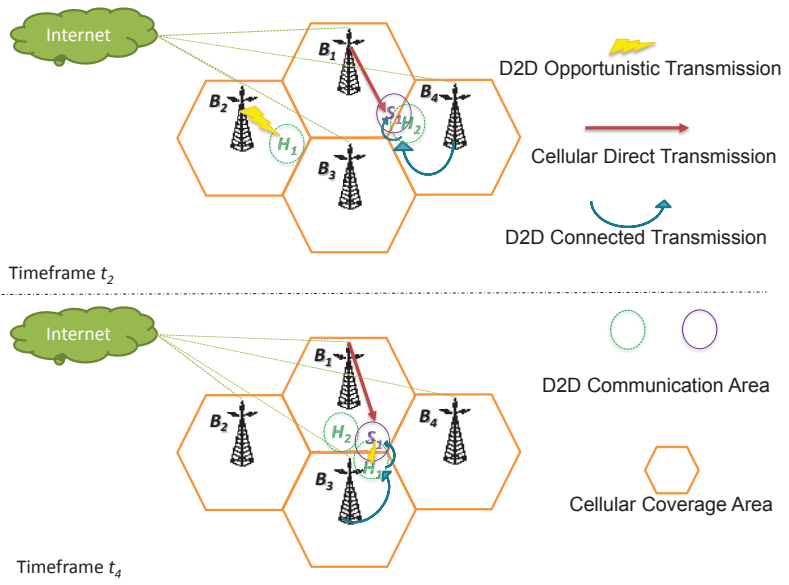

Fig. 1. Illustration of different transmission modes in D2D communication underlaying cellular network.

BSs, $B_{1}, B_{2}, B_{3}$ and $B_{4}$, which have high-speed Internet access and can distribute cellular resources to their associated UEs for cellular direct transmissions or D2D transmissions. In content-downloading, there are two natural groups of UEs, subscribers and helpers. Subscribers are the UEs that are requesting and downloading data, such as $S_{1}$ in Fig. 1 whose D2D communication area is denoted by small purple circle. The other group of the UEs who are not requesting data in the time period considered may act as helpers, such as $H_{1}$ and $H_{2}$ with green-circle D2D communication areas in Fig. 1. Although helpers may exhibit selfish behaviours, they can more or less participate in D2D transmissions by serving as "relays" via two possible D2D transmission modes introduced below.

1) D2D connected transmission: To take advantage of good local channel conditions and physical proximity of communicating UEs, a BS first transmits data flow to a voluntary helper and then requires the helper to immediately forward the messages it just received to a subscriber or another helper via a D2D link. In Fig. $1, B_{4}$ is transmitting data to $S_{1}$ with the aid of $\mathrm{H}_{2}$ during time frame $t_{2}$.

2) D2D opportunistic transmission: A helper who is willing to devote some buffer to D2D transmission can store and carry some data in its buffer and wait for an opportunistic contact to transmit the data to a subscriber or other helpers. In Fig. $1, B_{2}$ is transmitting data flow to $H_{1}$ for potential D2D opportunistic transmission during time frame $t_{2}$. Later, $H_{1}$ establishes D2D connection with $S_{1}$, and transmits the data from its buffer to it.

A user is required to contribute its battery resource in D2D connected transmissions, but both its battery and storage resources in D2D opportunistic transmissions. Therefore, we should consider different selfishness metrics for the two D2D communication modes. We divide both individual selfishness and social selfishness into two components, termed as connected selfishness and opportunistic selfishness, according to the above-mentioned two D2D transmission types.

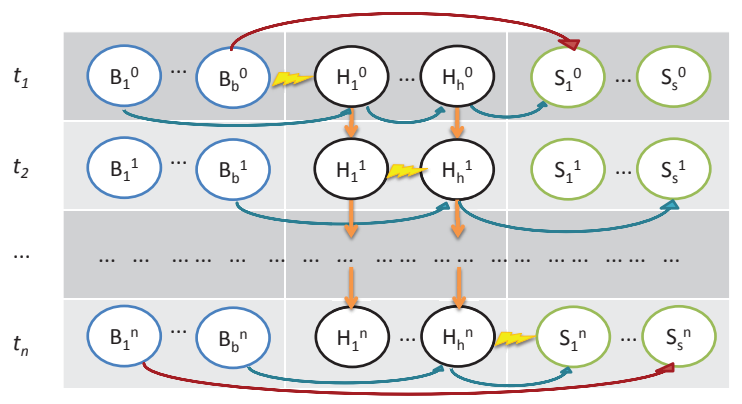

Fig. 2. Dynamic graph model which takes different types of selfishness into consideration and includes all possible data flow in D2D cellular system.

\section{MOdEL AND ANALYSIS FRAMEWORK}

To model this sophisticated scenario and analyze the impacts of both social and individual selfishness on D2D underlaying cellular networks, we propose an optimization framework that takes into account the two different D2D modes.

\section{A. Time-varying Graph Model and Objective}

If there are $b$ BSs labeled as $\mathcal{B}=\left\{B_{1}, \cdots, B_{b}\right\}, h$ helpers labeled as $\mathcal{H}=\left\{H_{1}, \cdots, H_{h}\right\}$ and $s$ subscribers labeled as $\mathcal{S}=\left\{S_{1}, \cdots, S_{s}\right\}$, a static graph similar to Fig. 1 with $b+$ $h+s$ nodes can be drawn for every time frame. We can use directed edges to model the data flows between nodes in a time frame. Specifically, the edges of outgoing D2D opportunistic flows are from willing helpers to subscribers or other helpers in a time frame as well as to themselves in the successive time frame, which represent the contents stored in the helpers' buffer. Similarly, the edges of D2D connected transmissions are from BSs via some voluntary helpers to subscribers, and the edges of cellular direct transmissions are directly from BSs to subscribers, all within a same time frame.

D2D opportunistic transmissions enable data flow across time frames and hence makes it possible to model the time evolution of this time-varying system by linking the static graphs of different time frames. Let the entire time period be divided into $n$ time frames. We first generate the $n$ graphs, one for each time frame, and then link them with directed edges to represent data flows in buffers across time frames. Fig. 2 includes all the possible transmission modes (cellular direct, D2D connected and D2D opportunistic transmissions), where BSs and UEs are represented by vertices. Moreover, directed edges are added to vertices, which represent the data flow of cellular direct transmission and/or D2D communication.

Next we attribute weights to directed edges to represent the magnitudes of data flows. Thus, each directed edge in the same row (time frame) in Fig. 2 is one of the following forms: crimson arrow for cellular direct transmission, blue arrow for D2D connected transmission, or yellow flash for D2D opportunistic transmission. Each edge is associated with a positive value representing the data flow transmitted. Furthermore, both incoming flow and outgoing flow of each helper in each time 
frame are divided into two flows - one for connected D2D transmission and one for opportunistic D2D transmission.

- Connected incoming flow: is the part of incoming flow of a helper for D2D connected transmission, and it will be forwarded immediately after reception and does not occupy the helper's buffer for inter-time frame transmission. The connected incoming flow of helper $H_{i}$ in time frame $l$ is denoted as $v_{l}\left(H_{i}\right)$.

- Opportunistic incoming flow: is the part of incoming flow of a helper for D2D opportunistic mode, and it will be stored in the helper's buffer for opportunistic transmission at some later time frames. The opportunistic incoming flow of helper $H_{i}$ in time frame $l$ is denoted as $w_{l}\left(H_{i}\right)$.

- Connected outgoing flow: is the part of outgoing flow that is just received and forwarded immediately by a helper. Connected outgoing flow equals to connected incoming flow for each helper in the graph model. The connected outgoing flow from helper $H_{i}$ to helper $H_{j}$ or subscriber $S_{j}$ in time frame $l$ is denoted as $x_{l}\left(H_{i}, H_{j}\right)$ or $x_{l}\left(H_{i}, S_{j}\right)$.

- Opportunistic outgoing flow: is the part of outgoing flow of a helper that is from the helper's buffer. The data in this kind of flow were received in previous time frames and have been stored in the helper's buffer. The opportunistic outgoing flow from helper $H_{i}$ to helper $H_{j}$ or subscriber $S_{j}$ in time frame $l$ is denoted as $y_{l}\left(H_{i}, H_{j}\right)$ or $y_{l}\left(H_{i}, S_{j}\right)$.

Note that connected and opportunistic selfishness impact connection states of each helper by imposing constraints on outgoing flows instead of incoming flows.

\section{B. Optimization Formulation and Solutions}

1) Optimization Object: Let the total data amount received by all the subscribers be $D$ in the time period considered. We can define the objective of our optimization framework as

$D=\sum_{l}\left\{\sum_{i, j}\left\{x_{l}\left(H_{i}, S_{j}\right)+y_{l}\left(H_{i}, S_{j}\right)\right\}+\sum_{k, j} c_{l}\left(B_{k}, S_{j}\right)\right\}$,

where the flow via cellular direct transmission from BS $B_{k}$ to node $S_{j}$ in time frame $l$ is denoted by $c_{l}\left(B_{k}, S_{j}\right)$.

2) Flow Conservation: In time frame $l$, the incoming flow via its own buffer (from previous time frame $l-1$ ) of helper $H_{i}$ is denoted as $a_{l}\left(H_{i}\right)$. At time frame $l=0$, the initial buffer of each helper is empty, i.e. $a_{0}\left(H_{i}\right)=0, \forall i$. We have the following constrains:

$$
\left\{\begin{array}{l}
y_{l}\left(H_{i}, H_{j}\right) \leq a_{l}\left(H_{i}\right), \forall i, j, l ; \\
y_{l}\left(H_{i}, S_{j}\right) \leq a_{l}\left(H_{i}\right), \forall i, j, l ; \\
w_{l}\left(H_{i}\right)+a_{l}\left(H_{i}\right) \geq a_{l+1}\left(H_{i}\right), \forall i, l ; \\
v_{l}\left(H_{i}\right)=\sum_{j} x_{l}\left(H_{i}, H_{j}\right)+\sum_{k} x_{l}\left(H_{i}, S_{k}\right), \forall i, l ; \\
\sum_{j}\left(y_{l}\left(H_{j}, H_{i}\right)+x_{l}\left(H_{j}, H_{i}\right)\right)+\sum_{k} c_{l}\left(B_{k}, H_{i}\right) \\
\quad=w_{l}\left(H_{i}\right)+v\left(H_{i}\right), \forall i, l .
\end{array}\right.
$$

3) Transmission rate and channel access: The transmission rate between each node pair must meet the resource constraint, and the total transmitted flow of each edge is constrained by the product of transmission rate and time-frame duration.
Moreover, considering connection states and user selfishness, the transmitted content flows must be strictly circumscribed within the connected and willing UEs in each time frame.

With the above-introduced constraints and optimization objective, a maximization problem can be formed, of which the decision variables include all the data flows. However, not all the associated constraints are linear constraints, and the problem does not belong to the category of linear programming problems. Fortunately, we use the reformulation linearization technique (RLT) [3] to transform those nonlinear constraints into linear expressions, and consequently use the existing optimization tool kits, such as CPLEX [7] and YALMIP [9], to solve the formulated maximization problem.

\section{ANALYSIS OF SELFISHNESS IMPACTS}

\section{A. Simulation Setup}

We utilize the proposed framework to analyze the impacts of selfishness on D2D underlaying cellular networks by the simulations using Cambridge trace [8], [10]. We use the method of [11] to compute the individual contact rates of node pairs by average statistics from the trace. We then average the contact rates of users in the same community as well as across communities to implement simulations. There are 35 realistic human mobility traces, among which 12 UEs are helpers. The total bandwidth is $20 \mathrm{MHz}$, and $80 \%$ of the cellular resources are allocated to BS for transmitting data to UEs, and the other $20 \%$ are used for D2D communications between UEs.

\section{B. Interaction between Individual Connected and Opportunis- tic Selfishness}

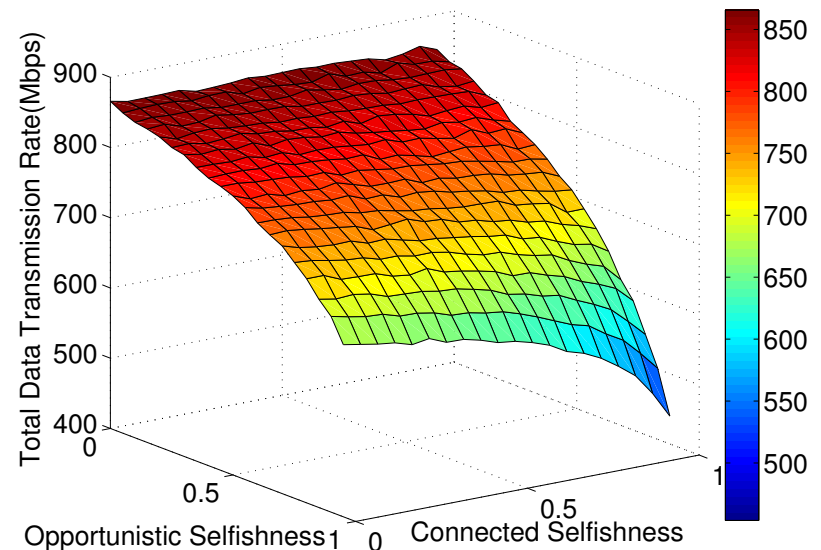

Fig. 3. The general trend of how total data transmission rate varies with two modes of individual selfishness.

We represent the two types of individual selfishness by two probabilities, individual connected selfishness probability (ICSP) and individual opportunistic selfishness probability (IOSP), which reflect helpers' unwillingness to cooperate in the two D2D modes, respectively. For example, if the ICSP is $c(0 \leq c \leq 1), c$ is the probability of a helper's refusal to cooperate with a UE within its D2D communication range in D2D connected communication. Fig. 3 depicts the trend of 


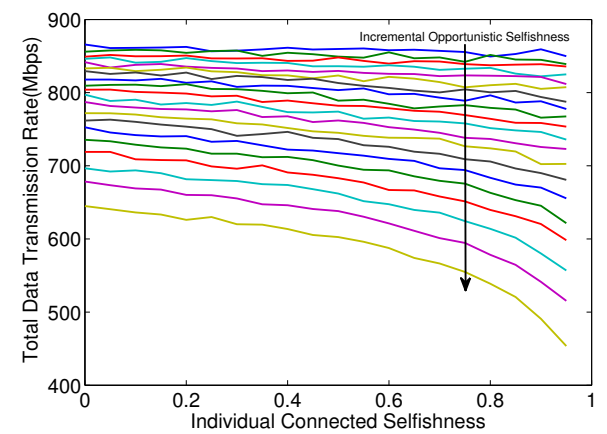

(a) Individual connected selfishness

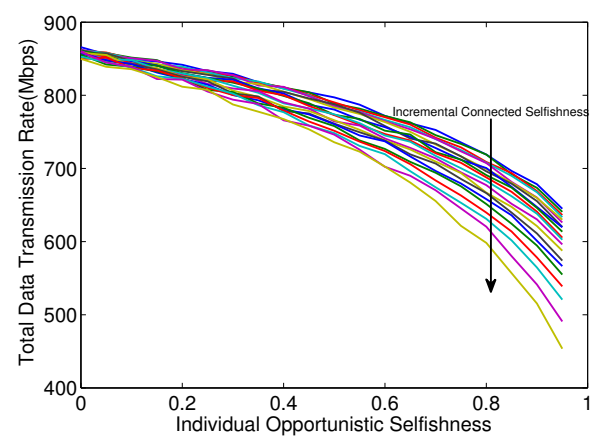

(b) Individual opportunistic selfishness

Fig. 4. The total transmission rate indicates an obvious interaction between two individual selfishness modes.

the total data transmission rate of all subscribers, influenced by the two selfishness. The results shows that a slight decrease in IOSP leads to a large increase in the data transmission rate, while ICSP has less influence on the system performance. An explanation is as follows. A helper with popular contents in its storage may transmit them during every D2D contact and, consequently, it can take the advantage of physical proximity more efficiently. Unfortunately, most users, left to their own will, are likely to increase IOSP instead of ICSP for the considerations of battery consumption, buffer occupation and potential hardware risks.

Intuitively, when ICSP is relatively low, the unoccupied cellular resources as the result of users' refusal to participate in D2D opportunistic transmission can be redistributed to the D2D connected transmission. Not surprisingly, Fig. 4 (a) and (b) indicate that the impacts of the D2D connected and D2D opportunistic selfishness are not independent. Denote the ICSP as $c$ and the IOSP as $o$. Further denote the total data transmission rate as $d=f(c, o)$, and its partial derivatives with respect to $c$ and $o$ as $p$ and $q$, respectively. We have

$$
p(c, o)=\frac{\partial f}{\partial c}(c, o)<0 \text { and } q(c, o)=\frac{\partial f}{\partial c}(c, o)<0 .
$$

Both $p$ and $q$ are approximately monotonically decreasing functions of $c$ and $o$, defined on the interval $[0,1]$. It indicates that with the increase of ICSP or IOSP, a same increment in IOSP or IOSP results in a sharper drop in the total data transmission rate. Therefore, if users are able to refuse D2D communication requests freely, the D2D communication system is likely to endure poor performance caused by high
D2D opportunistic selfishness.

Fortunately, there are possible solutions to this problem. If D2D connected mode is compulsory in the protocol, the results will regress to the highest (blue) curve in Fig. 4 (b), which shows that the dropped data transmission rate can be regained to some extend from around $600 \mathrm{Mbps}$ to around $700 \mathrm{Mbps}$ at high IOSP. By ensuring alternative D2D connected communication choices in the case of high rejection of D2D opportunistic mode, it makes the D2D communication underlaying cellular network resilient to high D2D opportunistic selfishness and avoids forcing users to devote their storage. Another possible solution is to set a minimum D2D-requestacceptance ratio and a reasonable buffer reservation, which constrains the system to the top left (the crimson part) of Fig. 3, and therefore guarantees a satisfying performance.

\section{Social Selfishness}

To reveal the impacts of social selfishness on D2D communications underlaying cellular networks, we use insidecommunity connected selfishness probability (IcC), outsidecommunity connected selfishness probability $(\mathrm{OcC})$, insidecommunity opportunistic selfishness probability (IcO) and outside-community opportunistic selfishness probability (OcO) to represent users' unwillingness to cooperate in the two

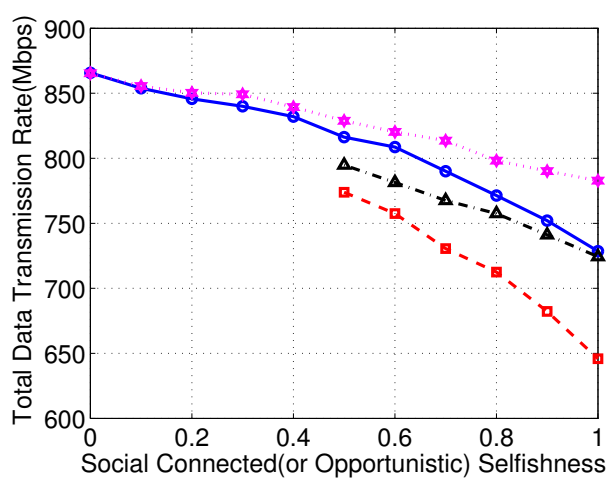

(a) Total data transmission rate

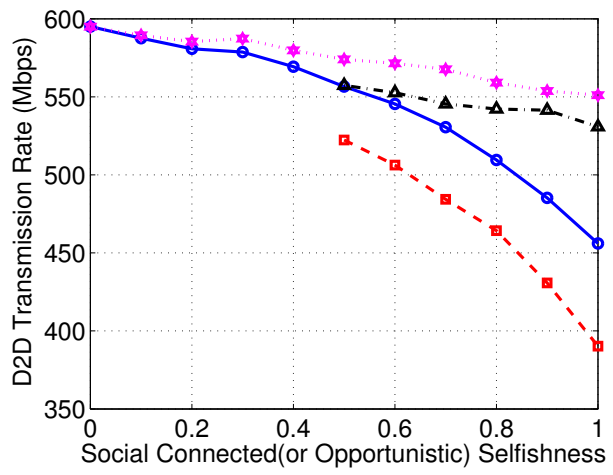

(b) D2D transmission rate

Fig. 5. System performance as a function of social selfishness with the four sets of representative selfishness parameters. 
D2D transmission modes with inside-community and outsidecommunity users, respectively. In the real world, it is reasonable to assume that $\mathrm{IcC} \leq \mathrm{OcC} \leq \mathrm{OcO}$ and $\mathrm{IcC} \leq \mathrm{IcO} \leq$ $\mathrm{OcO}$. After equally dividing the helpers and subscribers into two different social groups, we use the four different sets of $\mathrm{IcC}, \mathrm{OcC}, \mathrm{IcO}$ and $\mathrm{OcO}$ to perform simulations, and the results obtained are shown in Fig. 5, where the hexagrammarker curve models the inside-community-selfless users who are unwilling to devote buffer to strangers, and the trianglemarker curve models the users who are inside-communityselfless but very outside-community-selfish, while the circlemarker curves represents the users who are mainly concerned with buffer occupation, and the square-marker curve is for the users who are rather selfish. From the results of Fig. 5, we can draw the following observations.

1) Inside-Community and Outside-Community Selfishness: Comparing the circle-marker and square-marker curves in Fig. 5 (a) shows that a system consisted of the selfish users who are unwilling even to cooperate inside community ( $\mathrm{IcC}=0.5$ and $\mathrm{IcO}=0.5$ ) has a significantly degraded performance, compared with an inside-community-selfless system. By contrast, the gap between the hexagram-maker and circlemarker curves is relatively smaller, as the both systems consist of inside-community-selfless users. Outside-community selfishness has more impact on the D2D performance, as shown by the hexagram-maker and circle-marker curves of Fig. 5 (b). In the real-world, since users are more willing to cooperate with other inside-community users, we may reasonably assume low or even no inside-community selfishness, but the same cannot be said for outside-community selfishness.

2) Opportunistic and Connected Selfishness: From Fig. 5 (a), it can be seen that the gap between the hexagrammarker and circle-marker curves is smaller than that between the hexagram-marker and triangle-marker curves, indicating that $\mathrm{IcO}=0.5$ is more devastating than a larger OcC. Specifically, by focusing on the circle-marker and trianglemarker curves of Fig. 5 (a) with $\mathrm{OcC} \in[0.5,1]$, we observe that opportunistic selfishness causes larger degradation to the total data transmission rate than connected selfishness. But the reverse is true regarding the D2D data transmission rate, as can be seen by comparing the circle-marker and trianglemarker curves of Fig. 5 (b). An explanation is that for the system with high opportunistic selfishness and low connected selfishness (circle-marker one), more resource is allocated to cellular direct transmission. In fact, to optimize the total data transmission rate with high opportunistic selfishness, the system has to rely more on D2D connected communication with the aid of BS, which deprives the resource of cellular direct transmission. Thus, the results of Fig. 5 tell us that rather than forcing users to cooperate with strangers, service providers should make effort to persuade users to devote some of their storage to their own community.

3) Alleviated Interaction: If $\mathrm{IcC}=\mathrm{OcC}=\mathrm{IcO}=\mathrm{OcO}$, social selfishness will regress to individual selfishness. Specifically, these conditions specify the curve at which the plane $c=o$ cuts the surface in Fig. 3. In comparison to the results of individual selfishness, the circle-marker and square-maker curves in Fig. 5 (a) indicate less performance degradation especially when both selfishness modes are high. For example, in Fig. 5 (a), the lowest points of the circle-marker curve (insidecommunity selfless) and square-marker curve are approximately $730 \mathrm{Mbps}$ and $645 \mathrm{Mbps}$, respectively, yielding the worst performance degradation of $135 \mathrm{Mbps}$ and $220 \mathrm{Mbps}$, while the worst performance degradation in Fig. 3 is more than $400 \mathrm{Mbps}$. This indicates that a relatively low or little insidecommunity selfishness will neutralize to some extent the high outside-community selfishness, and consequently alleviates the performance degradation. Therefore, the results of Fig. 5 offers a potential solution to alleviate harmful interaction between the two selfishness modes - encouraging users to consider joint communities and to cooperate within their communities, which will benefit the whole D2D underlaying cellular system.

\section{CONCLusions}

In this paper, we have defined selfishness metrics for D2D communication underlaying cellular networks, according to the two D2D communication modes. We have also proposed an optimization framework for analyzing the impacts of both individual and social selfishness on the performance of D2D communications. Simulation results obtained under the realistic networking settings have indicated that the interaction between connected and opportunistic selfishness worsens the impairment caused by individual selfishness, while the harmful interaction caused by social selfishness can be alleviated.

\section{REFERENCES}

[1] L. Lei, Z. Zhong, C. Lin, and X. Shen, "Operator controlled deviceto-device communications in LTE-advanced networks," IEEE Wireless Commun., vol. 19, no. 3, pp. 96-104, Jun. 2012.

[2] G. Fodor, E. Dahlman, G. Mildh, S. Parkvall, N. Reider, G. Miklós, and Z. Turányi, "Design aspects of network assisted device-to-device communications," IEEE Commun. Mag., vol. 50, no. 3, pp. 170-177, Mar. 2012.

[3] B. Han, P. Hui, V. S. A. Kumar, M. V. Marathe, J. Shao, and A. Srinivasan, "Mobile data offloading through opportunistic communications and social participation," IEEE Trans. Mobile Computing, vol. 11, no. 5, pp. 821-834, May 2012.

[4] K. Doppler, M. Rinne, C. Wijting, C. B. Ribeiro, and K. Hugl, "Deviceto-device communication as an underlay to LTE-advanced networks," IEEE Commun. Mag., vol. 47, no. 12, pp. 42-49, Dec. 2009.

[5] N. Golrezaei, A. G. Dimakis, and A. F. Molisch, "Device-to-device collaboration through distributed storage," in Proc. Globecom 2012 (Anaheim, CA), Dec. 3-7, 2012, pp. 2397-2402.

[6] Y. Li, Z. Wang, D. Jin, and S. Chen, "Optimal mobile content downloading in device-to-device communication underlaying cellular networks," IEEE Trans. Wireless Commun., vol. 13, no. 7, pp. 3596-3608, Jul. 2014

[7] X. Chen, B. Proulx, X. Gong, and J. Zhang, "Social trust and social reciprocity based cooperative D2D communications," in Proc. MobiHoc'2013 (Bangalore, India), July 29 - Aug. 1, 2013, pp. 187-196.

[8] Y. Li, P. Hui, D. Jin, L. Su, and L. Zeng, "Evaluating the impact of social selfishness on the epidemic routing in delay tolerant networks," IEEE Commun. Letters, vol. 14, no. 11, pp. 1026-1028, Nov. 2010.

[9] N. Kayastha, D. Niyato, P. Wang, and E. Hossain, "Applications, architectures, and protocol design issues for mobile social networks: A survey," Proc. IEEE, vol. 99, no. 12, pp. 2130-2158, Dec. 2011.

[10] P. Hui, J. Crowcroft, and E. Yoneki, "Bubble rap: social-based forwarding in delay tolerant networks," in Proc. ACM MobiHoc, 2008, pp. 241250.

[11] W. Gao, Q. Li, B. Zhao, and G. Cao, "Multicasting in delay tolerant networks: a social network perspective," in Proc. ACM MobiHoc, 2009, pp. 299-308. 